\title{
Quantum Boltzmann statistics in interacting systems
}

\author{
Luigi Accardi, Sergei Kozyrev \\ January 25, 2020 \\ Centro Vito Volterra, Universita di Roma Tor Vergata
}

\begin{abstract}
Collective operators for generic quantum system with discrete spectrum are investigated. These operators, considered as operators in the entangled Fock space (space generated by action of collective creations on the vacuum) satisfy a particular kind of Quantum Boltzmann (or free) commutational relations.
\end{abstract}

\section{Introduction}

In the present paper we investigate the statistics of the interacting (or entangled) operators in the stochastic limit of quantum theory [1]. We investigate the model of quantum system interacting with reservoir (quantum field). The corresponding Hamiltonian is a combination of interacting, or entangled operators. Interacting operator is a product of operator of system and operator of reservoir, for example the product of annihilation of reservoir (bosonic quantum field) and a system operator

$$
D^{*} a(k)
$$

In the stochastic limit the quantum field becomes a quantum white noise. We will show that for the stochastic limit of discrete quantum system interacting with quantum field (for details see [2]) the statistics of entangled 
(or interacting) operators is the particular variant of Quantum Boltzmann, or free statistics. Analogous behavior (arising of free statistics) was already found for particular system Hamiltonian with continuous spectrum [3], [四], [0], [6].

In the present paper we will investigate generic discrete quantum system interacting with quantum field.

Definition. A quantum system with Hamiltonian $H_{S}$ acting in the Hilbert space $\mathcal{H}_{S}$ will be called generic, if:

i) The spectrum $S p e c H_{S}$ of the Hamiltonian is non degenerate.

ii) For any positive Bohr frequency $\omega>0$ there exists a unique pair of energy levels $\varepsilon_{1_{\omega}}, \varepsilon_{2_{\omega}} \in$ Spec $H_{S}$ such that:

$$
\begin{gathered}
H_{S}\left|1_{\omega}\right\rangle=\varepsilon_{1_{\omega}}\left|1_{\omega}\right\rangle, \quad H_{S}\left|2_{\omega}\right\rangle=\varepsilon_{2_{\omega}}\left|2_{\omega}\right\rangle \\
\omega=\varepsilon_{2_{\omega}}-\varepsilon_{1_{\omega}}>0
\end{gathered}
$$

The term generic means that the eigenvalues of $H_{S}$ are irregularly displaced. For example, the spectrum of the 1-dimensional harmonic oscillator satisfies (i) but not (ii). Thus it is not generic in the above sense.

In the stochastic limit, cf. [1], every positive Bohr frequency (a difference of eigenvalues of a system Hamiltonian) gives rise to a quantum white noise.

In the work [2] the authors showed that, in the stochastic limit, the dynamics of a (generic) quantum system is described by a stochastic Schrödinger equation which contains the following combination of quantum noises and system observables (that we call interacting, or entangled, or collective operator):

$$
c_{\omega}(t, k):=\left|2_{\omega}\right\rangle\left\langle 1_{\omega}\right| \otimes b_{\omega}(t, k)
$$

where the quantum white noise, cf. [1], satisfy the following (bosonic) relation

$$
\left[b_{\omega}(t, k), b_{\omega^{\prime}}\left(t^{\prime}, k^{\prime}\right)\right]=2 \pi \delta_{\omega \omega^{\prime}} \delta\left(t-t^{\prime}\right) \delta(\omega(k)-\omega) \delta\left(k-k^{\prime}\right)
$$

Actually even before the limit the evolution equation in the interaction picture contains terms of similar kind

$$
\left|2_{\omega}\right\rangle\left\langle 1_{\omega}\right| \otimes a(k) e^{-i t(\omega(k)-\omega)}
$$


This suggests that Skeide's analysis of the stochastic limit in terms of Hilbert modules [7] can be extended to the present case.

In the present paper we show that the operators (11) satisfy a variant of the Quantum Boltzmann (also called free, or infinite) commutation relations:

$$
a_{i} a_{j}^{*}=\delta_{i j}
$$

The Quantum Boltzmann (or free) algebra is generated by the $a_{j}, a_{k}^{*}$, called creation and annihilation operators, with the relations (3). No other relations are assumed and different creators $a_{i}^{*}, a_{j}^{*}$ do not commute. Therefore vectors in the Fock space of the type

$$
a_{i}^{*} a_{j}^{*}|0\rangle \neq a_{j}^{*} a_{i}^{*}|0\rangle
$$

are distinguishable and that justifies the name Quantum Boltzmann relations cf. for example [10], [11], [12]. Generalizations of such relations were found in the large $N$ limit of quantum chromodynamics [6], in models of particles interacting with a quantum field (which include quantum electrodynamics and the polaron model) [3], 四].

\section{Quantum Boltzmann statistics for entan- gled operators}

Given a generic quantum system, and a Bohr frequency $\omega$, denote $\left|1_{\omega}\right\rangle$ and

$\left|2_{\omega}\right\rangle$ the two eigenstates corresponding to the two energy levels, $\varepsilon_{1_{\omega}}, \varepsilon_{2_{\omega}}$, so that

$$
H_{S}\left|1_{\omega}\right\rangle=\varepsilon_{1_{\omega}}\left|1_{\omega}\right\rangle \quad, \quad H_{S}\left|2_{\omega}\right\rangle=\varepsilon_{2_{\omega}}\left|2_{\omega}\right\rangle \quad, \quad \omega=\varepsilon_{2_{\omega}}-\varepsilon_{1_{\omega}}>0
$$

With these notations we see that the restriction of $H_{S}$ on the space generated by $\left|1_{\omega}\right\rangle$ and $\left|2_{\omega}\right\rangle$ is

$$
\left(\begin{array}{cc}
\varepsilon_{2} & 0 \\
0 & \varepsilon_{1}
\end{array}\right)
$$

We define for each positive Bohr frequency $\omega$ the Pauli matrix that flips the spin down by

$$
\left|1_{\omega}\right\rangle\left\langle 2_{\omega}\right|=: \sigma_{\omega}^{-}=\left(\begin{array}{ll}
0 & 0 \\
1 & 0
\end{array}\right)
$$


Using this we define the interacting (or entangled, or collective) operator

$$
c_{\omega}(t, k):=\left|2_{\omega}\right\rangle\left\langle 1_{\omega}\right| \otimes b_{\omega}(t, k)=\sigma_{\omega}^{+} \otimes b_{\omega}(t, k)
$$

Remark. The operator (6) defined for non-positive Bohr frequency $\omega \leq 0$ is equal to zero, since the quantum white noise $b_{\omega}(t, k)$ for $\omega \leq 0$ equals to zero.

One can get the following relation on the collective operator, cf. 9]

$$
c_{\omega}^{2}(t, k)=0
$$

In the present paper we will investigate the case of many Bohr frequencies and get for collective operators the Quantum Boltzmann relations.

Remark. In the paper [9] operators of the form (6) were considered for the case of a single Bohr frequency and their vacuum statistics was shown to satisfy Boolean independence in the sense of von Waldenfels. The present paper extends this result to the case of multiple Bohr frequencies and establishes a connection between Boolean and Boltzmannian (or free) independence. It is interesting to notice that these types of independences arise naturally, i.e. from physically meaningful objects, in a purely bosonic context.

The dynamics of a system in the stochastic limit is described by the white noise (or master) Hamiltonian which in the considered case takes the form

$$
h(t)=\sum_{\omega \in F} \int d k \overline{g_{\omega}}(k) \sigma_{\omega}^{+} b_{\omega}(t, k)+\text { h.c. }
$$

in other words: if $H_{S}$ is generic, any generalized dipole interaction Hamiltonian of $S$ with a boson field is equivalent, in the stochastic limit, to a (possibly infinite) sum of independent 2-level-systems. The summation in (8) runs over the set of all Bohr frequencies. The simplest case corresponds to a single 2-level-system (one Bohr frequency), or the spin-boson model, cf. 8 .

Notice that the operators $\sigma_{\omega}^{ \pm}, b_{\omega}^{ \pm}$enter in the master Hamiltonian only through the combinations

$$
\sigma_{\omega}^{+} \otimes b_{\omega}(t, g) ; \quad \sigma_{\omega}^{-} \otimes b_{\omega}^{*}(t, g)
$$


and therefore the basic dynamical quantities like the propagator $U_{t}$, the wave operators $\Omega_{ \pm}$, the scattering operator $S$, will depend only on these combinations. This suggests to consider algebra generated by the entangled operators

$$
c_{\omega}(t, k):=\sigma_{\omega}^{+} \otimes b_{\omega}(t, k) ; \quad c_{\omega}^{*}(t, k):=\sigma_{\omega}^{-} \otimes b_{\omega}^{*}(t, k)
$$

then all the calculations involving only matrix elements of the iterated series expansion of $U_{t}$, the solution of the white noise Hamiltonian equation, cf. [1]

$$
\partial_{t} U_{t}=-i\left(\sum_{\omega \in F} \int d k \overline{g_{\omega}}(k) \sigma_{\omega}^{+} b_{\omega}(t, k)+\text { h.c. }\right) U_{t}
$$

with initial condition $U_{0}=1$, can be done entirely within this algebra.

A representation of the entangled algebra can be obtained within the Fock module $\mathcal{F}_{\text {ent }}$ which is the submodule of

$$
\mathcal{B}\left(\mathcal{H}_{S}\right) \otimes \mathcal{F}_{\text {mast }}
$$

where $\mathcal{F}_{\text {mast }}$ is the Fock space of the master field, $\mathcal{B}\left(\mathcal{H}_{S}\right)$ is the algebra of bounded operators in the system Hilbert space $\mathcal{H}_{S}$. The Fock module $\mathcal{F}_{\text {ent }}$ is a linear span of the entangled number vectors:

$$
\prod_{n} c_{\omega_{n}}^{*}\left(t_{n}, k_{n}\right)|0\rangle
$$

where $|0\rangle$ is the Fock vacuum of the master field.

Theorem. The operators $c_{\omega}(t, k), c_{\omega}^{*}(t, k)$ considered as operators on the Fock module $\mathcal{F}_{\text {ent }}$ of entangled number vectors satisfy the relations module

$$
c_{\omega}(t, k) c_{\omega^{\prime}}^{*}\left(t^{\prime}, k^{\prime}\right)=2 \pi \delta_{\omega \omega^{\prime}} \delta\left(t-t^{\prime}\right) \delta\left(k-k^{\prime}\right) \delta(\omega(k)-\omega) \sigma_{\omega}^{+} \sigma_{\omega}^{-}
$$

The operator $\sigma_{\omega}^{+} \sigma_{\omega}^{-}=\left|2_{\omega}\right\rangle\left\langle 2_{\omega}\right|$ in (10) is equal to the rank one orthogonal projector onto $\mathbf{C}\left|2_{\omega}\right\rangle$.

Remark. Notice that the operator $\sigma_{\omega}^{+} \sigma_{\omega}^{-}$in (10) conserves the entangled Fock space $\mathcal{F}_{\text {ent }}$.

Proof. Using the commutation relation (2) we get

$c_{\omega}(t, k) c_{\omega^{\prime}}^{*}\left(t^{\prime}, k^{\prime}\right)=2 \pi \delta_{\omega \omega^{\prime}} \delta\left(t-t^{\prime}\right) \delta\left(k-k^{\prime}\right) \delta(\omega(k)-\omega) \sigma_{\omega}^{+} \sigma_{\omega^{\prime}}^{-}+\sigma_{\omega}^{+} \sigma_{\omega^{\prime}}^{-} b_{\omega^{\prime}}^{*}\left(t^{\prime}, k^{\prime}\right) b_{\omega}(t, k)$ 
Let us show that the operator

$$
\sigma_{\omega}^{+} \sigma_{\omega^{\prime}}^{-} b_{\omega^{\prime}}^{*}\left(t^{\prime}, k^{\prime}\right) b_{\omega}(t, k)
$$

is equal to zero on the Fock module $\mathcal{F}_{\text {ent }}$. To check this we consider the action of (11) on a number vector of

$$
\prod_{n=1}^{l} c_{\omega_{n}}^{*}\left(t_{n}, k_{n}\right)|0\rangle
$$

The action of (11) on such a vector will be non-zero only if in the product (12) at least one of the creators is equal to $c_{\omega}^{*}(t, k)$. Let us denote $l_{0}$ the index of the first creator, starting from the left with this property. We get

$$
\sigma_{\omega}^{+} \sigma_{\omega^{\prime}}^{-} \prod_{1 \leq n<l_{0}} \sigma_{\omega_{n}}^{-} \sigma_{\omega}^{-} \prod_{l_{0}<n \leq l} \sigma_{\omega_{n}}^{-} \text {combination of } b_{\omega_{n}}^{*}\left(t_{n}, k_{n}\right)|0\rangle
$$

But for a generic system the combination

$$
\sigma_{\omega}^{+} \sigma_{\omega^{\prime}}^{-} \prod_{1 \leq n<l_{0}} \sigma_{\omega_{n}}^{-} \sigma_{\omega}^{-}=\left|2_{\omega}\right\rangle\left\langle 1_{\omega}\left|\sigma_{\omega^{\prime}}^{-} \prod_{1 \leq n<l_{0}} \sigma_{\omega_{n}}^{-}\right| 1_{\omega}\right\rangle\left\langle 2_{\omega}\right|
$$

equals to zero since

$$
\left\langle 1_{\omega}\left|\sigma_{\omega^{\prime}}^{-} \prod_{1 \leq n<l_{0}} \sigma_{\omega_{n}}^{-}\right| 1_{\omega}\right\rangle=0
$$

To prove this let us note that for generic Hamiltonian every operator $\sigma_{\omega_{k}}^{-}$ acting on eigenvector $\left|1_{\omega}\right\rangle$ of $H_{S}$ (with the eigenvalue $\varepsilon$ ) kills it or maps into the eigenvector $\left|2_{\omega}\right\rangle$ with the eigenvalue $\varepsilon-\omega$. Since arbitrary operator $\sigma_{\omega_{k}}^{-}$decreases the energy (this is exactly the place where the stochastic limit procedure is important, since in the stochastic limit only the terms with positive Bohr frequences in interaction Hamiltonian survive), and the product of $\sigma_{\omega_{k}}^{-}$in (13) is non-empty, the result of application of the product of $\sigma_{\omega_{k}}^{-}$ in (13) to $\left|1_{\omega}\right\rangle$ can be either zero or vector orthogonal to $\left|1_{\omega}\right\rangle$. Therefore the matrix element (13) is equal to zero.

We checked that the second term (11) vanishes that finishes the proof of relations (10).

Remark. One can find an alternative representation for the relations (10), defined by the operators

$$
c_{\omega}(t, k)=\sigma_{\omega}^{+} c(t) \otimes c_{\omega}(k)
$$


where $c(t), c_{\omega}(k)$ satisfy the relations

$$
\begin{gathered}
c(t) c^{*}\left(t^{\prime}\right)=\delta\left(t-t^{\prime}\right) \\
c_{\omega}(k) c_{\omega^{\prime}}^{*}\left(k^{\prime}\right)=2 \pi \delta(\omega(k)-\omega) \delta_{\omega \omega^{\prime}} \delta\left(k-k^{\prime}\right)
\end{gathered}
$$

This gives another representation of (10) using the tensor product of Quantum Boltzmann algebras. An analogous representation for the QED (quantum electrodynamics) entangled algebra was proposed in 4 . Moreover the operator $c_{\omega}(k)$ in (15) can be formally reproduced by the formula

$$
c_{\omega}(k)=c_{\omega} \otimes \sqrt{2 \pi \delta(\omega(k)-\omega)} c(k)
$$

where $c_{\omega}$ and $c(k)$ are Boltzmannian annihilators.

Remark. In the paper [5] the operator valued Quantum Boltzmann relations arising in the stochastic limit were called entangled, or interacting commutation relations.

Remark. In [5] a second quantized version of the module Quantum Boltzmann relations have been shown to be universal in any interaction with conservation of momentum.

The results of the present paper show that such relations arise in every generic open quantum system. This rises the problem of extending the above construction to non-generic systems thus obtaining a classification of all the possible commutation relations arising in the stochastic limit of quantum mechanics.

Remark. The term entanglement is usually referred to states and expresses the impossibility of representing a state vector of an open system as a tensor product, i.e. in the form

$$
\psi_{\text {system }} \otimes \psi_{\text {reservoir }}
$$

Such states are unstable under the evolution of interacting systems which creates linear combinations of them, i.e. states of the form:

$$
\sum_{n} \psi_{\text {system }}^{(n)} \otimes \psi_{\text {reservoir }}^{(n)}
$$

(with a number of terms strictly greater than 1) these are the entangled states. In the stochastic limit, the evolution of generic interacting systems 
(that creates entanglement) is described by Quantum Boltzmann relations of the type (10) for collective operators (system plus reservoir). Therefore we might say that entangled states present the Schrödinger picture of entanglement while the entangled relations correspond to the Heisenberg picture of entanglement.

\section{Acknowledgements}

The authors are grateful to I.V.Volovich and M.Skeide for discussions. Sergei Kozyrev is grateful to Centro Vito Volterra and Luigi Accardi for kind hospitality. This work was partially supported by INTAS 9900545 grant. Sergei Kozyrev was partially supported by RFFI 990100866 grant.

\section{References}

[1] Accardi, L., Lu, Y.G., Volovich, I.V., Quantum Theory and its Stochastic Limit, Springer Verlag, "Texts and Monographs in Physics" (2001)

[2] L.Accardi, S.V.Kozyrev, Lecture Notes of Volterra School on quantum interacting particles, Preprint of Centro Vito Volterra, 2000, Rome, also partially published in quant-ph/0005029

[3] Accardi, L., Lu, Y.G., Volovich, I.V., "Interacting Fock spaces and Hilbert module extensions of the Heisenberg commutation relations", Publications of IIAS, Kyoto, (1997).

[4] L.Accardi, S.V.Kozyrev, I.V.Volovich, Dynamical q-deformation in quantum theory and the stochastic limit, J.Phys.A, 32(1999) 3485-3495, q-alg/9807137

[5] L.Accardi, I.Ya.Aref'eva, I.V.Volovich, Non-Equilibrium Quantum Field and Entangled Commutation Relations, Special Issue of Proc. of Steklov Mathematical Institute dedicated to 90th anniversary of N.N.Bogoliubov

[6] I.Ya.Aref'eva, I.V.Volovich, Nucl. Phys. B462(1996), pp.600-615

[7] M. Skeide, Hilbert modules in quantum electro dynamics and quantum probability. Commun. Math. Phys. 192 (1998) 569-604 
[8] Accardi, L., Kozyrev, S.V., Volovich, I.V., "Dynamics of dissipative two-level system in the stochastic approximation", Phys.Rev.A 57, N3 (1997), quant-ph/9706021.

[9] M. Skeide, A central limit theorem for Bose Z-independent quantum random variables, Infinite Dimensional Analysis, Quantum probability and related topics, 2(1999) No.2, pp.289-300

[10] J. Cuntz, Commun.Math.Phys. 57 (1977) 173

[11] R.Speicher, Lett.Math.Phys. 27 (1993) 97

[12] D. Voiculescu, D. Dykema, A. Nica, Free Random Variables, CRM Monograph Series, Vol.1, American Math. Society, 1992 